\title{
Evaluation of central precocious puberty treatment with GnRH analogue at the Triangulo Mineiro Federal University (UFTM)
}

Maria de Fátima Borges', Priscila de Melo Franciscon', Thamy Contursi Cambraia', Débora Matias Oliveira', Beatriz Pires Ferreira', Elisabete Aparecida Mantovani Rodrigues de Resende', Heloísa Marcelina Cunha Palhares'

\begin{abstract}
Objective: To report our experience of treating central precocious puberty (CPP) with a GnRH analogue with respect to the final heights $(\mathrm{FH})$ attained in patients who completed treatment. Subjects and methods: Among 105 records of children diagnosed with precocious puberty, 62 cases $(54$ girls and 8 boys), who were treated with leuprolide acetate $/ 3.75 \mathrm{mg} / \mathrm{monthly}$, were selected, and divided into 4 groups: group 1 (G1), 25 girls who attained FH; group 2 (G2), 18 girls who completed treatment but did not reach FH; group 3 (G3), 11 girls still under treatment; and group 4 (G4), 8 boys, 5 of which attained FH. Treatment was concluded at a bone age of 12 years, and follow-up continued until FH was achieved. Results: In both G1 and G2 groups, height standard deviation score (SDS), weight-SDS and percentile of body mass index (PBMI) did not show intra/intergroup differences at the beginning and at interruption of treatment, but when added, G1+G2, height-SDS and weight-SDS differed significantly ( $p=0.002$ and 0.0001 , respectively). In $\mathrm{G} 1,19$ of 25 cases attained $T H$, and average height gain was $16.7 \mathrm{~cm}$ (7.7- 27.1); there was significant difference between $\mathrm{FH}$ and prediction of $\mathrm{FH}$ at the start (PFH at start) $(p=0.0001)$, as well as between PFH at interruption vs TH and vs FH $(p=0.007)$ with $\mathrm{FH}$ higher than $\mathrm{TH}(\mathrm{p}=0.004)$. Significant correlation was identified between $\mathrm{FH}$ and height gain after treatment. Conclusion: As shown by some studies, $\mathrm{GnRH}$ analogue treatment was effective in children with CPP reaching FH near the genetic target. Arch Endocrinol Metab. 2015;59(6):515-22
\end{abstract}

Keywords

GnRH analogue; final stature; target stature; precocious central idiopathic puberty; precocious puberty

' Disciplina de Endocrinologia,

Universidade Federal do Triângulo Mineiro (UFTM), Uberaba, MG, Brasil

Correspondence to: Maria de Fátima Borges Praça Dr. Thomaz Ulhôa, 50 38025-050 - Uberaba, MG, Brasil borgmf@uol.com.br

Received on Jun/25/2015 Accepted on Jul/27/2015

DOI: 10.1590/2359-3997000000101

\section{INTRODUCTION}

G $\mathrm{nRH}$ analogues have been available for treating central precocious puberty (CPP) since the 1980 s, initially as nasal and subcutaneous preparations given every 8 hours $(1,2)$. In the late 1980s, long-acting preparations for monthly intramuscular administration (3) and, more recently, medications of quarterly application and annual subcutaneous implants were developed $(4,5)$.

This medication is expensive and is supplied by the State Secretariats of Health of several states in Brazil by means of a protocol attesting diagnosis of CPP at an age when treatment will be beneficial (6). The discipline of Endocrinology at UFTM, abiding by the conditions of the treatment and proper completion of the protocols, has indicated and received $\mathrm{GnRH}$ analogue for monthly use since 1995 from the State Secretariat of Health of the State of Minas Gerais. The adminis- tration of the medication has been centralized in Belo Horizonte (the State capital city) and is performed by a team appointed for this purpose.

After accumulating experience in the use of this medication, we retrospectively evaluated the results of the treatment with a GnRH analogue in children of both genders with CPP, aiming to analyze clinical and laboratory aspects. We also intended to observe the final height attained by patients who completed treatment, comparing it with the genetic target and the initial height predictions.

\section{SUBJECTS AND METHODS}

A total of 105 medical records of children classified as CPP between 1991 and 2014 (January), were selected from the archives of the Discipline of Endocrinology. After an initial analysis, 25 were excluded because they had been treated with medroxyprogesterone be- 
fore treatment with $\mathrm{GnRH}$ analogue, which became available in 1995. Another 18 children with a confirmed diagnosis of CPP were also excluded; 10 did not comply with the treatment, and 8 had co-morbidities interfering with growth, resulting in 62 patients available for analysis. These patients included 54 girls and 8 boys, and they were divided into 4 groups. Group 1 (Gl) consisted of 25 girls who completed the treatment and reached a final height. Group 2 (G2) comprised 18 girls, who completed the treatment and are still being followed but not yet reached a final height; Group 3 (G3) comprised 11 girls who are still receiving treatment. Group 4 (G4) comprised 8 boys, and three of them are still being treated; the other 5 achieved a final height. All children met the following inclusion criteria: 1. presence of secondary sex characteristics before the age of 8 years for girls and 9 years for boys; 2 . increase in growth velocity and advanced bone age; 3 . release of the hypothalamic-pituitary-gonadal axis demonstrated by hormone tests showing stimulated values of $\mathrm{LH}$ and FSH, obtained by a GnRH test; 4. imaging exams, computerized tomography or nuclear magnetic resonance of the skull, in addition to pelvic ultrasound in the girls, allowing classification by etiology into idiopathic CPP (ICPP) or secondary to lesions of the central nervous system; 5 . monthly IM administration of longacting GnRH analogue as the only treatment for CPP.

Although this study was retrospective, in this outpatient clinic, the children were evaluated according to diagnostic and treatment protocols $(7,8)$, including anthropometric data such as weight and height, and the respective standard deviations of their means (Z-scores or SDS), body mass index (BMI) and percentile distribution of BMI (PBMI), which were calculated and plotted into curves standardized for Brazilian children $(9,10)$. Bone age was obtained based on an X-ray of the left hand, evaluated according to the method developed by Greulich and Pyle (11). The target or genetic target height was also calculated, using the conventional formula [TH: (mother's height + father's height) $/ 2+6.5$ (boys) and -6.5 (girls)]. Predicted final height $(\mathrm{PFH})$ was calculated using average Bayley \& Pinneau tables (12) to compare data at three time points: at the beginning, at the interruption of the GnRH analogue and at menarche. The stage of sexual development was classified according to Marshall and Tanner $(13,14)$. As part of the medical attention routine, the basal and $\mathrm{GnRH}$ stimulated concentrations of LH and FSH and sexual steroids (estradiol in girls and testosterone in boys) were measured by immunochemiluminescence (ICMA) but only stimulated concentrations of $\mathrm{LH}$ were considered in the diagnosis of CPP. When the release of the hypothalamic-pituitary-gonadal axis (peak of $\mathrm{LH}>4.0 \mathrm{IU} / \mathrm{mL}$ after acute $\mathrm{GnRH}$ ) was observed, within a clinical context, its blockage was indicated (15).

Effectiveness of treatment was evaluated every 6 months, based on a set of clinical and laboratory data such as growth velocity, non-progress of bone age and pubertal stage, basal concentrations of LH and estradiol (in girls) and of testosterone (in boys), as well as $\mathrm{LH}$ peak after i.v. GnRH $\leq 2.3 \mathrm{IU} / \mathrm{L}(16,17)$.

The following variables were also analyzed: 1 . Final height $(\mathrm{FH})$ : measured when the patient's growth in the last 2 years was less than $1 \mathrm{~cm} /$ year and when bone age was more than 16 years. All the patients were summoned, and their height was measured. 2. M-I growth: difference (in $\mathrm{cm}$ ) between height at menarche and height at the interruption of the treatment; 3 . FH-I growth: difference (in $\mathrm{cm}$ ) between final height and height at interruption of treatment, which was considered height gain post treatment; 4. FH-TH: difference $(\mathrm{cm})$ between final height attained and target height; 5 . $\Delta$ Bone Age-C. Age: difference between bone age and chronological age; 6 . Time to beginning of treatment: the time between the first symptom mentioned by the mother (telarche or pubarche) and the first appointment and until the beginning of treatment.

\section{Statistical analysis}

Data distribution was parametric according to the Kolmogorov-Smirnov and Levene's variance homogeneity tests. Comparison of more than two variables was performed by analysis of variance (ANOVA), followed by Tukey's test. The data obtained before and after treatment were analyzed using Student's paired $t$ test. At the tables, the test used in each comparison performed was indicated. Values of $\mathrm{p}<0.05$ were considered significant. Correlations between height gain since interruption of treatment until achievement of final height (growth FH-I), as well as final height and anthropometric variables, target height and height predictions, were evaluated by Pearson's test.

\section{RESULTS}

At the first appointment, patient ages in group l (Gl) varied from 1.3 to 8.6 years (mean: $6.9 \pm 1.7$ ) (Table 1 ). 
They were treated for a period of time varying from 0.6 to 6.2 years (mean: $2.1 \pm 1.5$ ). In group $2(\mathrm{G} 2)$, patients at the first appointment were between 5.2 and 8.4 years old (mean: $7,3 \pm 0,9$ ) (Table 1 ). They were treated for a period of time varying from 0.7 to 4.6 years (mean: $1.8 \pm 1.0)$. In group $3(\mathrm{G} 3)$, patients were first seen at ages varying from 3.7 to 7.8 years (mean: $6.7 \pm 1.7$ ) (Table 1).

The boys in group 4 (G4), consisted of children aged from 3.9 to 8.3 years $(5.7 \pm 2.3)$ at the first appointment and, due to the low number, they were not represented in tables. In this group, 2 had hypothalamic hamartomas, 2 had hydrocephaly, 1 had a $3^{\text {rd }}$ ventricle cyst, and 3 had idiopathic CPP. Three are still being treated, and the other 5 achieved a final height. This group was characterized by fast growth, advanced bone age and final height below the target height $(167.0 \pm$ $8.5 \mathrm{~cm}$ vs $170.6 \pm 8.5 \mathrm{~cm})$. Two patients reached and exceeded the target height. However, the small number of patients does not allow for a statistical comparison with the female group.

All female patients (G1, G2 and G3) had idiopathic central precocious puberty, and their clinical and laboratory data are presented in tables 1,2 and 3 . Although some patients were seen at very young ages, most of them only sought specialized treatment at around the age of 7 years, yet reported onset of telarche between 5 and 6 years, characterizing a delay in evaluation and specialized treatment. This delay was assessed by calculating the time interval between the first pubertal symptom mentioned by the mother until the first clinical appointment and was $0.9 \pm 0.8$ years (minimum: 0.1 - maximum: 3.0$), 0.8 \pm 0.4$ years $(0.2-1.5)$, and $0.5 \pm 0.7$ years $(0.2-1.2)$ in G1, G2 and G3, respectively. This time interval was calculated also, as the time between the first pubertal symptom until the beginning of $\mathrm{GnRH}$ treatment and was $1.8 \pm 0.8$ years $(0.7-3.5)$, $1.6 \pm 1.3$ years $(0.2-6.3)$ and $1.6 \pm 0.6$ years $(0.2-$ $1.5)$ in G1, G2 and G3, respectively. The clinical and anthropometric data of the three groups at the beginning of treatment are presented in table 1 and did not show any significant difference between the 3 groups. The laboratory measurements regarding hormone levels, which confirmed the diagnosis of precocious puberty (PP), are also presented in table 1 , showing a similar LH peak in the 3 groups.

The female patients reached the previously described treatment effectiveness patterns without needing any adjustment of the initial GnRH analogue doses. In the male group, however, 2 patients with hypothalamic hamartoma had to have their GnRH analogue doses doubled during the treatment.

Table 1. Clinical data at the beginning of follow-up of girls with precocious puberty

\begin{tabular}{lccc}
\hline Clinical data & G1 (n: 25) & G2 (n: 18) & G3 (n: 11) \\
\hline C. Age $^{1}(\mathrm{yr})$ & $6.9 \pm 1.7(1.3-8.6)^{2}$ & $7.3 \pm 0.9(5.2-8.4)$ & $6.7 \pm 1.7(3.7-7.8)$ \\
Telarche (yr) & $5.1 \pm 1.8(0.6-7.9)$ & $6.3 \pm 1.4(2.0-7.8)$ & $6.3 \pm 1.8(3.5-7.6)$ \\
Pubarche (yr) & $6.4 \pm 1.9(0.6-9.6)$ & $7.3 \pm 1.1(5.7-10.0)$ & $6.7 \pm 0.9(5.8-7.6)$ \\
Height SDS & & $1.27 \pm 1.14(-0.14 \rightarrow 4.10)$ & $0.14 \pm 0.87(-1.36 \rightarrow 0.83)$ \\
Weight SDS & $1.05 \pm 1.03(-0.80 \rightarrow 2.58)$ & $1.17 \pm 1.1(-0.78 \rightarrow 2.81)$ & $0.28 \pm 1.44(-1.92 \rightarrow 2.04)$ \\
PBM| ${ }^{4}$ & $0.95 \pm 1.15(-0.33 \rightarrow 4.06)$ & $70.67 \pm 25.28(10.0-99.0)$ & $63.74 \pm 37.14(6.7-99.0)$ \\
Bone Age & $71.56 \pm 22.71(20.0-98.0)$ & $8.8 \pm 2.1(5.0-12.0)$ & $7.8 \pm 2.1(4.3-10.0)$ \\
$\Delta$ Bone Age-C. Age (yr) & $8.3 \pm 2.3(2.0-12.0)$ & $1.5 \pm 1.3(0.1-3.6)$ & $1.2 \pm 0.9(0.2-2.6)$ \\
Target Height (cm) & $1.7 \pm 1.1(0.2-4.3)$ & $159.0 \pm 4.3(153.0-170.0)$ & $156.0 \pm 4.8(151.0-162.0)$ \\
Basal LH (IU/L) & $158.0 \pm 5.1(147.0-170.0)$ & $1.65 \pm 3.12(0.10-13.12)$ & $0.71 \pm 0.76(0.12-2.03)$ \\
Basal FSH (IU/L) & $0.92 \pm 0.98(0.15-4.60)$ & $2.99 \pm 1.53(0.77-6.78)$ & $4.20 \pm 2.16(1.09-7.20)$ \\
Estradiol (pg/mL) & $2.52 \pm 1.90(0.30-7.57)$ & $32.00 \pm 17.27(13.70-72.00)$ & $28.96 \pm 23.46(5.00-58.50)$ \\
LH peak (IU/L) & $27.00 \pm 22.79(6.50-114.00)$ & $22.61 \pm 41.58(4.00-183.09)$ & $13.42 \pm 6.72(7.12-21.28)$ \\
LH/FSH & $15.87 \pm 22.14(4.00-107.00)$ & $0.98 \pm 1.34(0.02-4.27)$ & $0.17 \pm 0.16(0.05-0.45)$ \\
\hline
\end{tabular}

${ }^{1}$ C. Age: chronological age expressed in years (yr).

${ }^{2}$ Minimum and maximum.

${ }^{3}$ SDS: standard deviation score.

${ }^{4}$ PBMI: percentile distribution of body mass index.

${ }^{5} \Delta$ Bone age-C. Age: difference between bone age and chronological age.

ANOVA: for inter-group comparisons of all represented data: G1 vs G2 vs G3 $=p>0.05$.

G1: group treated that already attained the final height; G2: group treated that did not attain the final height yet; G3: group receiving treatment. 
Table 2. Clinical data of female patients with idiopathic central precocious puberty (ICPP) at the beginning of treatment and at 3 time points of treatment evaluation

\begin{tabular}{|c|c|c|c|}
\hline Data analyzed & G1 (n: 25) & G2 (n: 18) & G1+G2 (n: 43) \\
\hline \multicolumn{4}{|l|}{ Beginning of treatment } \\
\hline C. Age $(y r)^{1}$ & $7.5 \pm 1.5(3.3-9.0)^{2}$ & $7.9 \pm 1.0(5.3-9.3)$ & $7.7 \pm 1.3(3.3-9.3)$ \\
\hline Height SDS ${ }^{3}$ & $1.20 \pm 1.08(-0.41 \rightarrow 2.56)$ & $1.44 \pm 1.19(-0.55 \rightarrow 4.05)$ & $1.32 \pm 1.13(-0.55 \rightarrow 4.05)$ \\
\hline Weight SDS & $1.12 \pm 1.23(-0.19 \rightarrow 4.54)$ & $1.56 \pm 1.40(-0.35 \rightarrow 4.86)$ & $1.34 \pm 1.32(-0.35 \rightarrow 4.86)$ \\
\hline $\mathrm{PBMI}^{4}$ & $72.6 \pm 24.3(15.0-97.0)$ & $75.9 \pm 22.8(18.0-99.0)$ & $74.2 \pm 23.3(15.0-99.0)$ \\
\hline Bone Age (yr) & $9.5 \pm 2.5(3.0-12.0)$ & $9.8 \pm 1.9(5.0-13.0)$ & $9.3 \pm 2.3(3.0-13.0)$ \\
\hline$\Delta$ Bone Age-C.Age $(y r)^{5}$ & $1.8 \pm 1.3(0.1-3.9)$ & $1.4 \pm 1.2(0.1-3.5)$ & $1.7 \pm 1.3(0.1-3.9)$ \\
\hline \multicolumn{4}{|l|}{ Interruption of treatment } \\
\hline C. Age & $9.8 \pm 0.5(9.0-11.1)$ & $9.8 \pm 0.5(9.2-11.3)$ & $9.8 \pm 0.5(9.0-11.3)$ \\
\hline Height SDS & $1.51 \pm 0.96(-0.54 \rightarrow 3.06)$ & $1.74 \pm 1.11(-0.85 \rightarrow 3.85)$ & $1.61 \pm 1.02(-0.85 \rightarrow 3.85)$ \\
\hline Weight SDS & $1.47 \pm 1.32(-0.33 \rightarrow 4.25)$ & $2.29 \pm 1.48(-0.24 \rightarrow 4.73)$ & $1.82 \pm 1.43(-0.33 \rightarrow 4.73)$ \\
\hline PBMI & $71.4 \pm 24.3(15.0-98.0)$ & $83.4 \pm 23.6(18.0-98.0)$ & $76.3 \pm 24.4(15.0-98.0)$ \\
\hline Bone Age & $11.8 \pm 1.3(10.0-15.0)$ & $11.9 \pm 0.7(11.0-13.0)$ & $11.9 \pm 1.0(10.0-15.0)$ \\
\hline$\triangle$ Bone Age-C. Age & $2.3 \pm 1.2(0-4.7)$ & $2.3 \pm 0.8(0.6-3.8)$ & $2.3 \pm 1.0(0-4.7)$ \\
\hline \multicolumn{4}{|l|}{ Menarche } \\
\hline C. Age & $11.3 \pm 1.2(9.3-13.3)$ & $10.6 \pm 0.5(10.0-11.4)$ & $11.0 \pm 1.0(9.3-13.3)$ \\
\hline Height SDS & $1.69 \pm 1.06(0.01 \rightarrow 3.00)$ & $1.38 \pm 0.90(-0.07 \rightarrow 2.59)$ & $1.58 \pm 0.99(-0.07 \rightarrow 3.00)$ \\
\hline Weight SDS & $1.63 \pm 1.68(0.34 \rightarrow 6.00)$ & $1.58 \pm 1.38(0.26 \rightarrow 4.10)$ & $1.61 \pm 1.55(0.26 \rightarrow 6.00)$ \\
\hline BMIP & $69.7 \pm 27.7(24.0-99.0)$ & $72.5 \pm 30.2(23.0-99.0)$ & $70.6 \pm 27.9(23.0-99.0)$ \\
\hline Bone Age & $12.9 \pm 1.0(12.0-15.0)$ & $12.7 \pm 1.3(11.0-15.0)$ & $12.8 \pm 1.2(11.0-15.0)$ \\
\hline$\triangle$ Bone Age-C. Age & $1.8 \pm 1.5(0-5.0)$ & $2.3 \pm 1.5(0.5-5.0)$ & $2.0 \pm 1.5(0-5.0)$ \\
\hline \multicolumn{4}{|l|}{ Final evaluation } \\
\hline C. Age when summoned & $18.8 \pm 2.5(15.0-22.0)$ & $11.7 \pm 1.3(10.1-14.5)$ & \\
\hline Final Height (FH) & $162.0 \pm 6.2(150.0-174.0)$ & - & \\
\hline Weight & $61.0 \pm 16.0(42.3-118.5)$ & - & \\
\hline BMI & $23.0 \pm 5.2(16.5-41.0)$ & - & \\
\hline Bone Age & Adult & $13.3 \pm 1.4(11.0-15.0)$ & \\
\hline
\end{tabular}

${ }^{1}$ C. Age: chronological age, all ages expressed in years (yr).

2 minimum and maximum.

${ }^{3}$ SDS: standard deviation score.

${ }^{4}$ PBMI: percentile distribution of body mass index.

${ }^{5} \triangle$ Bone age- $C$. Age: difference between bone age and chronological age in years.

ANOVA: for intra-group comparisons in G1 and G2.

[height SDS, weight SDS, PBMI, $\triangle$ Bone Age-C. Age (beginning vs interruption vs menarche)] $p>0.05$.

Student's paired t test: for intra-group comparisons before and after treatment.

$\mathrm{G} 1+\mathrm{G} 2$ [height SDS (initial vs interruption)] $\mathrm{p}=0.002$.

$\mathrm{G} 1+\mathrm{G} 2$ [weight SDS (initial vs interruption)] $\mathrm{p}=0.0001$

$\mathrm{G} 1+\mathrm{G} 2$ [PBMI (initial vs interruption)] $p>0.05$.

In table 2, the clinical data of interest are displayed at 3 time points: 1 . at the beginning of the treatment; 2. at interruption of the treatment; 3 . at menarche; in Gl, clinical data at the recording of the final height $(\mathrm{FH})$ is also included. Initially, in G1 and G2, an intra-group statistical comparison of anthropometric data obtained at these 3 time points was performed. Then, these 2 groups were associated and the data common to both were analyzed (i.e., until interruption of treatment). No significant difference was found in either group regarding the anthropometric data (height SDS and weight SDS, PBMI and $\Delta$ Bone Age-C. Age). When G1 and G2 were associated $(\mathrm{Gl}+\mathrm{G} 2)$, a significant difference was found in the comparison of the initial height SDS vs interruption $(\mathrm{p}=0.002)$ and also in the comparison of the initial weight SDS vs interruption $(\mathrm{p}=0.001)$, but there was no difference in PBMI. 
Table 3. Prediction of final height at the beginning (PFH at start), at interruption of treatment and at menarche, height gain from interruption until menarche (M-I growth) in group 1 (G1) and 2 (G2), and until final height (FH-I growth) in G1

\begin{tabular}{lcc}
\hline Comparisons & G1 (n: 25) & G2 (n: 18) \\
\hline PFH at start $(\mathrm{cm})^{1}$ & $155.0 \pm 9.0$ & $162.0 \pm 9.0$ \\
& $(141.0-171.0)^{2}$ & $(150.0-185.0)$ \\
PFH at interruption & $160.0 \pm 8.5$ & $161.0 \pm 7.0$ \\
& $(147.0-176.0)$ & $(149.0-177.0)$ \\
PFH at menarche & $161.0 \pm 7.6$ & $157.0 \pm 8.2$ \\
& $(149.0-173.0)$ & $(142.0-165.0)$ \\
Treatment duration $(\mathrm{yr})$ & $2.1 \pm 1.5(0.6-6.2)$ & $1.8 \pm 1.0(0.7-4.6)$ \\
M-I growth $(\mathrm{cm})$ & $10.2 \pm 6.2(0.3-18.7)$ & $7.2 \pm 4.6(1.7-14.0)$ \\
FH-I growth $(\mathrm{cm})$ & $16.7 \pm 5.1(7.7-27.1)$ & - \\
Final height $(\mathrm{FH})$ & $162.0 \pm 6.2$ & - \\
& $(150.0-174.0)$ & \\
Target height $(\mathrm{TH})$ & $158.0 \pm 5.1$ & $159.0 \pm 4.3$ \\
& $(147.0-170.0)$ & $(153.0-170.0)$ \\
FH-TH & $4.08 \pm 6.63$ & - \\
& $(-13.0 \rightarrow 16.0)$ & \\
\hline
\end{tabular}

${ }^{1}$ All heights measured in $\mathrm{cm} ;{ }^{2}$ minimum and maximum.

ANOVA: for intra-group comparisons:

G1 and G2 [PFH (at start vs at interruption vs at menarche)], $p>0.05$

$\mathrm{G} 1+\mathrm{G} 2=[\mathrm{PFH}$ (at start vs at interruption vs at menarche) $], \mathrm{p}>0.05$.

$\mathrm{G} 1+\mathrm{G} 2=[\mathrm{PFH}$ (at start vs at interruption vs at menarche )], $\mathrm{p}>0.05$

G1 (PFH at interruption vs TH vs FH), ANOVA, $\mathrm{p}=0.007$.

Student's $t$ test:

G1 and G2 [PFH (at start; at interruption; at menarche) vs TH], $p>0.05$.

G1 (PFH at start vs FH) $p=0.0001$.

$\mathrm{G} 1$ (TH vs FH) $p=0.004$.

Prediction of final height (PFH) in G1 and G2 was also evaluated at the beginning of treatment, at its interruption or at menarche (Table 3 ). No intra or inter-group significant difference was found at these 3 time points. Additional data are presented in table 3: treatment duration, which was greater in Gl because of already completing the treatment; height gain from interruption of treatment to menarche (M-I growth) was $10.2 \pm 6.2 \mathrm{~cm}$ in G1 and $7.2 \pm 4.6 \mathrm{~cm}$ in G2, but these data could not be compared because $40 \%$ of the G2 girls did not yet presented menarche; height gain from interruption of treatment to $\mathrm{FH}(\mathrm{FH}-\mathrm{I})$ was 16.7 \pm 5.1 in Gl; $\mathrm{FH}$ and $\mathrm{FH}-\mathrm{TH}$ in Gl, and yet in this group, comparison between the initial $\mathrm{PFH}$ vs $\mathrm{TH}$ vs $\mathrm{FH}$ showed a significant difference $(\mathrm{p}=0.007)$, with $\mathrm{FH}$ exceeding the TH $(\mathrm{p}=0.004)$ and the initial PFH $(\mathrm{p}=0.0001)$. Analyzing the patients individually, in G1, 16/25 patients not only reached but actually exceeded the target height, whereas $3 / 25$ attained the exact TH. From a statistical perspective, the differences were shown after the interruption of treatment when the pubertal growth spurt had already occurred.
To study the possible factors that could have influenced the height gain from interruption of treatment until final height (FH-I growth) and FH, we performed correlations of these two clinical parameters with anthropometric data at the beginning and at interruption of treatment, as well as $\mathrm{TH}$, treatment duration, and estradiol concentration at the beginning of treatment, as this last one is considered to be an important causal factor in the advance of the bone age (Table 4). An inverse and significant correlation was identified between FH-I growth and chronological age at the beginning and at interruption of treatment, and with bone age at interruption. A positive correlation was found between $\mathrm{FH}$ and height SDS at the beginning of treatment and at its interruption, as well as between the $\mathrm{FH}$ and height gain from interruption of treatment (Table 4).

Table 4. Pearson's correlations between height gain from interruption (I) of treatment until final height (FH), (FH-I growth) and between $\mathrm{FH}$ and some data of interest, in the group 1

\begin{tabular}{|c|c|c|c|c|}
\hline \multirow[t]{2}{*}{ Data evaluated } & \multicolumn{2}{|c|}{ (FH-I growth) } & \multicolumn{2}{|c|}{ FH } \\
\hline & $r$ & p & $r$ & $\mathbf{p}$ \\
\hline \multicolumn{5}{|l|}{ Beginning of treatment } \\
\hline C. Age $^{1}$ & -0.403 & $0.045^{\star}$ & -0.230 & 0.267 \\
\hline Bone Age & -0.389 & 0.054 & -0.303 & 0.140 \\
\hline$\triangle$ Bone Age-C. Age ${ }^{2}$ & -0.248 & 0.230 & -0.240 & 0.246 \\
\hline Height SDS ${ }^{3}$ & -0.123 & 0.556 & 0.570 & $0.003^{*}$ \\
\hline Target height & 0.342 & 0.093 & 0.290 & 0.158 \\
\hline Estradiol & -0.313 & 0.127 & -0.030 & 0.884 \\
\hline Treatment duration & 0.154 & 0.462 & 0.103 & 0.624 \\
\hline \multicolumn{5}{|c|}{ Interruption of treatment } \\
\hline C. Age & -0.622 & $<0.0001^{\star}$ & -0.243 & 0.241 \\
\hline Bone Age & -0.500 & $0.0125^{\star}$ & -0.136 & 0.516 \\
\hline$\triangle$ Bone Age-C. Age & -0.200 & 0.336 & -0.037 & 0.860 \\
\hline Height SDS & -0.006 & 0.976 & 0.825 & $<0.0001^{*}$ \\
\hline FH-I growth & - & - & 0.433 & $0.030^{*}$ \\
\hline
\end{tabular}

${ }^{1} \mathrm{C}$. Age: chronological age.

${ }^{2} \triangle$ Bone age $-C$. Age: difference between bone age and chronological age.

${ }^{3}$ SDS: standard deviation score.

* Statistically significant.

\section{DISCUSSION}

Children receiving care at the public health care system at the Triangulo Mineiro School of Medicine (Sistema Único de Saúde/UFTM) have had access to GnRH analogues for the treatment of CPP since 1995, when they started receiving this medication from the Minas Gerais State Health Secretariat, based on an analysis of 
protocols sent to the State Capital, Belo Horizonte. The entire State of Minas Gerais still suffers from this limitation (i.e., the decision to release the medication is not up to the child's treating physician, but depends on a centralized team), adding insecurity and delay in the beginning of treatment.

Although the mean times elapsed between the first symptom, the first doctor's appointment and start of treatment have improved, there are still bureaucratically enforced difficulties to overcome. In 2007, Lazar and cols. (18) treated 115 girls with a GnRH analogue; 22 were diagnosed and began treatment at the age of 6 years, 38 at 6 to 8 years, and 55 at 8 to 9 years (considered early matured). The residual growth predicted at the interruption of treatment was attained only in the 22 girls diagnosed and treated early (i.e., before the age of 6 years). Therefore, the prognosis of height is better with earlier diagnosis and treatment onset.

Although height is an important issue and constitutes a main objective, this treatment also intended to preserve the time of childhood, readjusting the child hormonally and psychically to his or her age range and peers $(7,17)$. Thus, a reduction in growth velocity and involution in the Tanner breast growth stage is observed, with menarche postponed to a more acceptable age $(16,19)$.

In the present study, of the total 62 girls, 11 are still receiving treatment with a $\mathrm{GnRH}$ analogue, whereas 43 already completed treatment. Of these, 25 reached the $\mathrm{FH}$, checked after calling the patients in for evaluation. Some of the data could only be obtained in this latter group. After treatment interruption, many patients lose the motivation to return to the clinic to measure height and weight, even every six months. During the summoning process, our team had to actively search for the patients, requiring a social worker.

The initial data showed no differences between Gl and G2 (Table 1), demonstrating the same pattern of patients coming to the service. At the three time points chosen for the analysis of patients who completed the treatment (Table 2), we found no differences when we evaluated the patients of G1 and G2, but when we combined the two groups $(\mathrm{Gl}+\mathrm{G} 2)$, the height SDS was significantly higher at treatment interruption $(\mathrm{p}=$ $0.002)$, whereas the bone age showed no significant change (Table 2).

The weight SDS $(\mathrm{G} 1+\mathrm{G} 2)$ also showed differences $(\mathrm{p}=0.0001)$ between the beginning and the interruption of treatment, whereas the PBMI remained stable
(Table 2). Individual evaluation of the patients revealed that $17(39.5 \%)$ already had a PBMI compatible with overweight/obesity at the beginning of the treatment. At treatment interruption, this number rose to $24 \mathrm{pa}-$ tients $(55.8 \%)$. However, of the patients summoned for evaluation of the $\mathrm{FH}(\mathrm{n}=25)$, only $7(28 \%)$ presented with overweight/obesity. Some studies have been calling attention to a higher prevalence of overweight/obesity as a side effect of the treatment with GnRH analogue (20-22), but our data suggest that this association is found only if the nutritional status (PBMI) is used as an evaluation parameter at treatment interruption and not over the long term during which a decrease is observed.

The height predictions at the several time points did not differ from each other, and the $\mathrm{FH}$ was significantly higher than any predicted height and higher than the TH (Table 3), indicating limitations of the mathematical models in front of the biological variability as suggested by some studies (23-26).

Between treatment interruption and menarche, Gl displayed a mean height gain of $10.2 \pm 6.2 \mathrm{~cm}(0.3-$ $18.7 \mathrm{~cm}$ ) (Table 3), indicating the occurrence of the pubertal growth spurt within this period of time and showing that there were children who grew as much as $18.7 \mathrm{~cm}$ and children whose menarche occurred almost immediately after interruption of growth. From treatment interruption until confirmation of the $\mathrm{FH}$, there was a mean height gain of $16.7 \pm 5.1 \mathrm{~cm}(7.7$ $-27.1 \mathrm{~cm})$, indicating that a mean residual growth of $6.5 \mathrm{~cm}$ occurs after menarche, with great variability. In G2, in which the FH has not yet been reached, the figures showed a similar pattern (Table 3 ). The mean post-treatment height gain obtained in Gl $(7.7-27.1$ $\mathrm{cm}$ ) was greater than that reported by Lazar and cols. in a patient group analyzed prior to the age of 6 years (treated early). A possible explanation for this result is that, in our patients, the $\mathrm{FH}$ was obtained at the mean age of 18.8 years ( $15-22$ years), when the individuals had certainly completed their growth.

Among the factors that positively affected the $\mathrm{FH}$, we identified the height SDS at the beginning of treatment $(\mathrm{r}=0.700, \mathrm{p}=0.003)$ and at interruption $(\mathrm{r}$ $=0.825, \mathrm{p}<0.0001)$, in addition to growth after interruption of treatment $(\mathrm{r}=0.433, \mathrm{p}=0.030)$ (Table 4 ). These data suggest that children who are taller at the beginning of treatment will be taller at the end, and that the growth spurt after treatment interruption was important for reaching the final height, as previously demonstrated by other authors (27-29). However, 
growth after treatment interruption presented an inverse and significant correlation with chronological age at the start and end of treatment and with bone age at the end of treatment (Table 4), indicating that the time of beginning and interrupting treatment can modify the final result. We discontinued the treatment at a chronologic age of $9.8 \pm 0.5$ years and a bone age of 11.8 \pm 1.3 years (in G1, but G2 is similar - Table 2), and menarche occurred at a chronologic age of $11.3 \pm 1.2$ years, oftentimes under parental protests, as they would have liked to postpone it for at least another year. Our data suggest that, although the decision to discontinue treatment has to be made individually, a chronologic age of approximately 10 years and a bone age of approximately 12 years may represent a "window of opportunity" for good height gain after treatment interruption.

In 2 boys in the male group who had hypothalamic hamartoma, the clinical and hormonal control was somewhat more difficult, as they had accelerated growth velocity and bone age as well as earlier sexual maturation, with lack of suppression of the hypothalamic-pituitary-gonadal axis. These factors required adjustment of GnRH to higher doses.

In the reviewed classical literature on this subject (23-30), the patients are either limited to girls or if patients includes boys with CPP, their number is much smaller and is rather due to organic conditions. To enable comparisons with ICPP, multicenter studies such as Mul and cols. are necessary, where 26 boys with ICPP from 3 different countries were evaluated and final heights close to the target height were reported (31).

Hormonal control was also helpful in cases of undesirable behavior tending toward aggressiveness, impulsivity or sexual precociousness, playing an important role in the child's social insertion, as demonstrated by the literature $(7,17)$.

The limitations of the present study are the retrospective design, the size of the cohort, and the difficulties of a long term longitudinal follow-up study. These characteristics prevent definitive conclusions, but, as has been shown by other authors (16-18,24,26), treatment with $\mathrm{GnRH}$ analogues was effective in the clinical and hormonal control of children with CPP, primarily in girls, being a fundamental means to make them reach a FH compared to TH. Our data also suggest that diagnosis and beginning of treatment should be earlier, as these factors can interfere with the final height. Additionally, the appropriate time for discontinuing the treatment must be detected to allow adequate height gain.
Acknowledgments: Zilda Cristina dos Santos - collaborating social worker and Adriana Paula da Silva, collaborating nutritionist from Triangulo Mineiro Federal University (UFTM).

Fundings: this research was supported by UFTM.

Disclosure: no potential conflict of interest relevant to this article was reported.

\section{REFERENCES}

1. Ash RH, Balmaceda JP, Neves de Castro M, Shally AV. Comparison of the subcutaneous and intranasal administration of an $\mathrm{LH}$ $\mathrm{RH}$ antagonist ([N-Ac-D-p-Cl-Phe1,2,D-Trp3,D-Arg6,D-Ala10]-LH$\mathrm{RH}$ ) in the rhesus monkey. Adv Contracept. 1985;1(2):109-17.

2. Rime JC, Zumsteg V, Blumberg A, Hadzislimovic F, Gerard J, Zurbrügg RP. Long-term treatment of central precocious puberty with an intranasal LHRH analogue: control of pituitary function by urinary gonadotropins. Eur J Pediatric. 1988;147(3):263-9.

3. Roger $\mathrm{M}$, Chaussain JL, Berlier $\mathrm{P}$, Bost $\mathrm{M}$, Canlorbe $\mathrm{P}$, Colle $\mathrm{M}$, et al. Long term treatment of male and female precocious puberty by periodic administration of a long-acting preparation of D-Trp6-luteinizing hormone-releazing hormone microcapsules. J Clin Endocrnol Metab. 1986;62(4):670-7.

4. Mericq V, Lammoglia JJ, Unanue N, Villaroel C, Hernández MI, Ávila A, et al. Comparison of three doses of leuprolide acetate in the treatment of central precocious puberty: preliminary results. Clin Endocrinol (Oxf). 2009;71(5):686-90.

5. Eugster EA, ClarkeW, Kleter GB, Lee PA, Weely K, Reiter EO, et al. Efficacy and safety of histrelin subdermal implant in children with central precocious puberty: a multicenter trial. J Clin Endocrinol Metab. 2007;92(5):1697-704.

6. Protocolos Clínicos e Diretrizes Terapêuticas do Ministério da Saúde Volume I- Puberdade Precoce Central. Portaria SAS/MS ${ }^{\circ}$ 111, de 23 de abril de 2010. Available from: bvsms.saude.gov.br.

7. Carel JC, Eugster EA, Rogol A, Ghizzoni L, Palmert MR; on behalf of the members of the ESPE-LWPES GnRH Analogues Consensus Conference Group. Consensus statement on the use of gonadotropin-releasing hormone analogs in children. Pediatrics. 2009;123(4):752-62.

8. Macedo DB, Cukier P, Mendonça BB, Latronico AC, Brito VN. Advances in the etiology, diagnosis and treatment of central precocious puberty. Arq Bras Endocrinol Metab. 2014;58(2):108-17.

9. Marcondes E, Berquó E, Hegg R, Colli AS, Zacchi MAS. Crescimento e Desenvolvimento Pubertário em Crianças e Adolescentes Brasileiros. I - Metodologia. São Paulo: Editora Brasileira de Ciências; 1982.

10. Marcondes RM, Marcondes E, Berquó E, Prandi R, Yunes J. Crescimento e Desenvolvimento Pubertário em Crianças e Adolescentes Brasileiros. II - Altura e Peso. São Paulo: Editora Brasileira de Ciências; 1982.

11. Greulich WW, Pyle S. Radiographic atlas of skeletal development of the hand and wrist. 2nd Ed. Stanford CA: Stanford University Press; 1959.

12. Bayley N, Pinneau S. Tables for predicting adult height from skeletal age: revised for use with the Greulich-Pyle hand standards. J Pediatr. 1952;40(4):423-41.

13. Marshall WA, Tanner JM. Variation in pattern of pubertal changes in girls. Arch Dis Child.1969;44(235):291-303.

14. Marshal WA, Tanner JM. Variations in pattern of pubertal in boys. Arch Dis Child. 1970;45(239):13-23.

15. Resende EA, Lara BH, Reis JD, Ferreira BP, Pereira GA, Borges MF. Assessment of basal and gonadotropin-releasing-hormone- 
stimulated gonadotropins by immunochemiluminometric and immunofluorometric assays in normal children. J Clinic Endocrinol Metabol. 2007;92(4):1424-9.

16. Brito VN, Latronico AC, Arnhold IJ, Mendonça BB. A single luteinizing hormone determination 2 hours after depot leuprolide is useful for therapy monitoring of gonadotropin-dependent precocious puberty in girls. J Clin Endocrinol Metab. 2004;89(9):4338-42.

17. Kim YJ, Lee HS, LeeYJ, Lim JS, Kim EY, Jin DK et al. Multicenter clinical trial of leuprolid acetate depot (Luphere depot $3.75 \mathrm{mg}$ ) for efficacy and safety in girls with central precocious puberty. Ann Pediatr Endocrinol Metab. 2013;18(4):173-8.

18. Lazar L, Padoa A, Phillip M. Growth pattern and final height after cessation of gonadotropin-suppressive therapy in girls with central sexual precocity. J Clin Endocrinol Metab. 2007;92(9):3483-9.

19. Carel JC, Léger J. Precocious puberty. N Engl J Med. 2008;358(22):2366-77.

20. Palmert MR, Mansfield MJ, CrowleyWF Jr, Crigler JF Jr, Crawford JD, Boepple PA. Is obesity an outcome of gonadotropin-releasing hormone agonist administration? Analysis of growth and body composition in 110 patients with central precocious puberty. J Clin Endocrinol Metab. 1999;84(12):4480-8.

21. Taşcilar ME, Bilir P, Akıncı A, Köse K, Akçora D, Inceoğlu D, et al. The effect of gonadotropin-releasing hormone analog treatment (leuprolide) on body fat distribution in idiopathic central precocious puberty. Turk J Pediat. 2011;53(1):27-33.

22. Colmenares A, Gunczler P, Lanes R. Higher prevalence of obesity and overweight without an adverse metabolic profile in girls with central precocious puberty compared to girls with early puberty, regardless of $\mathrm{GnRH}$ analogue treatment. Int J Pediatr Endocrinol. 2014;2014(1):5.

23. Oostdijk W, Drop SL, Odink RJ, Hummelink R, Parsch CJ, Sippell WG. Long-term results with a slow-release gonadotrophinreleasing hormone agonist in central precocious puberty. DutchGerman Precocious Puberty Study Group. Acta Paediatr Scand Suppl. 1991;372:39-45.
24. Klein KO, Barnes KM, Jones JV, Feuillan PP, Cutler GB Jr. Increased final height in precocious puberty after long-term treatment with LHRH agonists: the National Institutes of Health experience. J Clin Endocrinol Metab. 2001;86(10):4711-6.

25. Weise M, Flor A, Barnes KM, Cutler GBJr, Baron J. Determinants of growth during gonadotropin-releasing hormone analog therapy for precocious puberty. J Clin Endocrinol Metab. 2004;89(1):103-7.

26. Pasquino AM, Pucarelli I, Accardo F, Demiray V, Segni M, Di Nardo L. Long-term observation of 87 girls with idiopathic central precocious puberty treated with gonadotropin-releasing hormone analogs: impact on adult height, body mass index, bone mineral content, and reproductive function. J Clin Endocrinol Metab. 2008;93(1):190-5.

27. Brito VN, Latronico AC, Cukier P, Teles MG, Silveira LFG, Arnhold IJP, et al. Factors determining normal adult height in girls with gonadotropin-dependent precocious puberty treated with depot gonadotropin-releasing hormone analogs. J Clin Endocrinol Metab. 2008;93(7):2662-9.

28. Benetti-Pinto CL, Camargo LBN, Magna LA, Garmes HM, Petta CA. Predictive factors for height gain in idiopathic central precocious puberty treated with $\mathrm{GnRH}$ analogues. Rev Bras Ginecol Obstetr. 2008;30(12):609-13.

29. Carel JC, Roger M, Ispas S, Tondu F, Lahlou N, Blumberg J, et al. Final height after long-term treatment with triptorelin slow release for central precocious puberty: importance of statural growth after interruption of treatment. French study group of Decapeptyl in Precocious Puberty. J Clin Endocrinol Metab. 1999;84(6):1973-8.

30. Lee PA, Klein K, Mauras N, Lev-VaislerT, Bacher P. 36-month treatment experience of two doses of leuprolide acetate 3-month depot for children with central precocious puberty. J Clin Endocrinol Metab. 2014;99(9):3153-9.

31. Mul D, Bertelloni S, Carel JC, Saggese G, Chaussain JL, Oostdijk W. Effect of gonadotropin-releasing hormone agonist treatment in boys with central precocious puberty: final height results. Horm Res. 2002;58(1):1-7. 